\title{
Heart Transplantation
}

\author{
Takeshi Nakatani, MD
}

\begin{abstract}
A total of 59 heart transplantations (HTx) have been performed in Japan as of September, 2008, since the Organ Transplantation Law was settled in October 1997. The majority of recipients were suffered from dilated cardiomyopathy and waiting condition of all recipients were status 1 . The mean waiting time was 777 day; 50 patients $(85 \%)$ were supported by several types of left ventricular assist systems (LVAS) and the mean duration of support was 780 days. The majority of patients underwent operation by modified bicaval method with Celsior solution for cardiac preservation, and $64 \%$ of recipients were administered triple therapy with cyclosporine, mycophenolate mofetil, and steroid as the initial immunosuppressive regimen. The 9-year survival rate was $94 \%$, which was superior to that of the international registry. HTx in Japan has been very limited by a severe shortage of donors, but the results have been excellent even though the majority of recipients were waiting for long-term with a LVAS as a bridge to HTx. (Circ J 2009; Suppl A: A-55-A-60)
\end{abstract}

Key Words: Bridge to heart transplantation; Heart transplantation; Left ventricular assist systems

$\mathbf{T}$ he first human-to-human heart transplantation (HTx) was performed in South Africa on December 3, $1967^{1}$ and by the end of 1968, 102 HTx had been performed in 52 institutions. However, the results were disappointing, with $60 \%$ early mortality and a mean survival of only 29 days 2 During the 1970 s, a few institutions continued to perform HTx. The transvenous endomyocardial biopsy technique with a histologic system of grading was developed for monitoring heart allograft rejection, and in the early $1980 \mathrm{~s}$, the introduction of cyclosporine for immunosuppression improved the results of HTx. Following this progress, the number of HTx procedures increased dramatically and it has become the standard option for end-stage heart failure. According to the international registry, more than 80,000 HTx have been performed worldwide to date?

In Japan, the Organ Transplantation Law was settled in October 1997, and first HTx under this law was performed in 1999. Since then, a total of 59 HTx have been performed in Japan as of September 2008. From May 2001, HTx for dilated cardiomyopathy (DCM) and the dilated phase of hypertrophic cardiomyopathy ( $\mathrm{dHCM})$ was approved as highly advanced medical technology. Enforcement of HTx performed in institutes selected by the Joint Committee of Transplant-related Societies was covered by health insurance from April 2006.

\section{Listing and Waiting for HTx}

HTx is considered a therapeutic option for the patient with advanced heart disease that is life-threatening and refractory to the traditional medical and/or surgical therapies. Major heart diseases are DCM, dHCM and ischemic heart disease (IHD). Of those, DCM and dHCM should be diagnosed by

(Received May 13, 2009; accepted May 15, 2009; released online June 5, 2009)

Department of Organ Transplantation, National Cardiovascular Center, Suita, Japan

Mailing address: Takeshi Nakatani, MD, Department of Organ Transplantation, National Cardiovascular Center, 5-7-1 Fujishiro-dai, Suita 565-8565, Japan. E-mail: tnakatan@hsp.ncvc.go.jp

All rights are reserved to the Japanese Circulation Society. For permis-

sions, please e-mail: cj@j-circ.or.jp endomyocardial biopsy.

If patients fulfill the following conditions, they are candidates for HTx.

1. Require prolonged or repeated hospitalization.

2. Continuing to be New York Heart Association class 3-4 despite traditional therapy including $\beta$-blockers and angiotensin-converting enzyme inhibitors.

3. Life-threatening severe arrhythmia that is uncontrollable with traditional antiarrhythmic treatment.

4. Under 60 years of age (desirable).

The following conditions render them unsuitable.

1. Severe non-cardiac disease (eg, severe dysfunction of liver or kidney, significant obstructive pulmonary disease, malignancy, severe autoimmune disease, collagen disease, etc).

2. Active peptic ulcer or infection, severe diabetes mellitus, excessive obesity.

3. Tobacco, alcohol or drug abuse.

4. Psychoneurosis.

5. Fixed pulmonary vascular resistance greater than 6 Wood units.

6. HIV antibody positive.

In the investigation of HTx candidacy, a therapeutic approach excepting HTx, predicted life expectancy, and compliance with post-transplant treatment should be considered carefully because medical therapy including immunosuppression is necessary throughout the patient's lifetime. In Japan, determination of HTx candidates has 2 phases. Heart transplant committees of the Japanese Circulation Society as well as each institution examine the HTx indications. When the patient is accepted as a HTx candidate by both committees, informed consent of the candidate and family is obtained at each HTx institution. After that, the candidate is enrolled in the Japan Organ Transplant Network (JOTNW). HTx operations are now limited to 6 institutes chosen by the Joint Committee of Transplant-related Societies.

Treatment for heart failure continues while waiting for HTx. In some cases, native heart function will recover enough to quit the waiting list. In other cases, candidacy will be lost because of severe organ dysfunction, infection or various disorders. Therefore, review of candidacy is performed every 6 months. When heart failure progresses and 


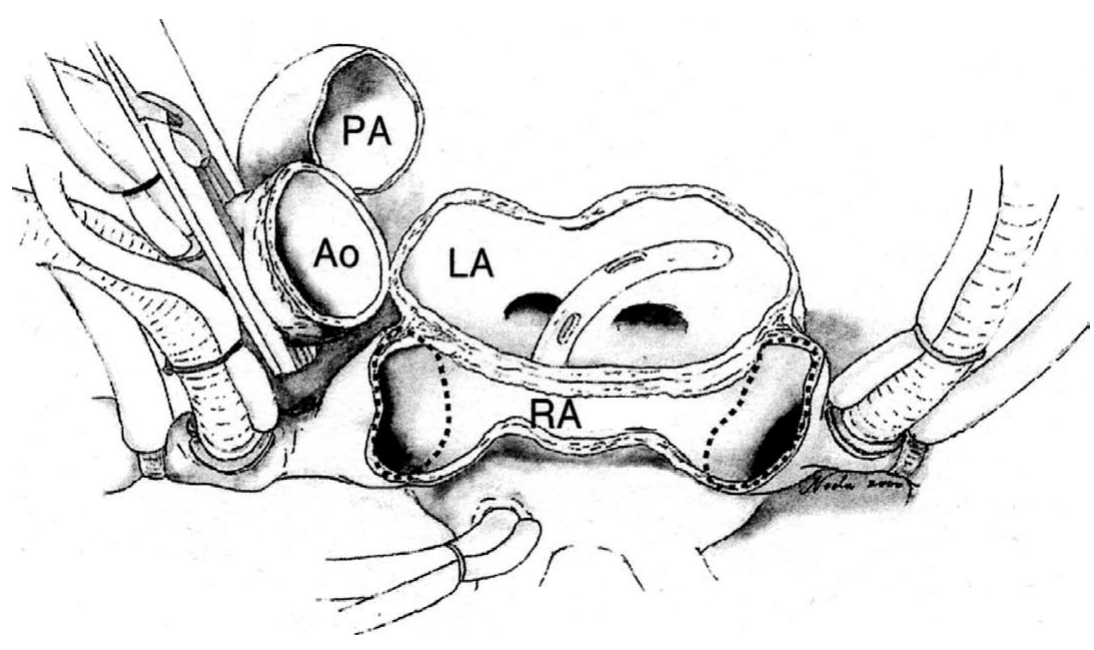

Figure 1. Modified bicaval method6 PA, pulmonary artery; Ao, aorta; LA, left atrium; $\mathrm{RA}$, right atrium. organ function deteriorates, application of a ventricular assist system (VAS) will be considered as a bridge-to-transplantation. At present, the Toyobo-National Cardiovascular Center type VAS is used under health insurance. Several types of implantable left VAS (LVAS) are applied as clinical trials. Many listed cases are supported with a VAS before listing or while waiting for HTx in Japan.

\section{Examination of Donor Heart and Selection of Candidate for HTx}

First, the appropriateness of the donor for organ transplantation is reviewed. If there is active systemic infection, positivity for HIV antibody, HTLV-1 antibody, hepatic B surface antigen or HCV antibody, diagnosis or suspicion of Creutzfeld-Jakob disease, or malignant tumor, the donor is unsuitable. Subsequently, examination as a donor for HTx is performed. If there is diagnosed heart disease, cardiac trauma or a history of open-heart surgery, the donor is unsuitable. As a donor for HTx, age less than 60 years is desirable; in men over 45 years old or women over 50 years old, consideration must be given to coronary arteriosclerosis. Cardiac condition should be carefully examined by electrocardiography and echocardiography, especially when large doses of inotropic agents have been used $(>10 \mu \mathrm{g}$. $\mathrm{kg}^{-1} \cdot \mathrm{min}^{-1}$ of dopamine equivalency). Final judgment is confirmed by visual inspection and palpation of the donor heart.

A candidate for recipient is chosen from the waiting list of the JOTNW when the potential donor is considered suitable. Selection from the list is according to the following policy: matching blood type (identical/compatible), size (body weight between $80 \%$ and $130 \%$ of the donor is suitable), and negative in direct cross-match. When several candidates are selected, the maximum period of donor heart ischemia before operation within $4 \mathrm{~h}$, status of waiting (priority to status 1 candidates), and the waiting time. As status 1 , consideration is given to (1) supported by VAS, (2) supported by IABP, (3) under ventilator support, and (4) continuous infusion of inotropes treated in the intensive care rooms such as ICU or CCU.

\section{Transplant Operation}

One of the key issues in HTx is donor selection and management of the donor. The ideal cardiac donor is of a suitable age with stable hemodynamics. Management to stabilize hemodynamics is important for expanding the donor pool. Cause of death, cardiac arrest and cardiopulmonary resuscitation, chest trauma, and medical history, especially cardiovascular disease, are checked. Hemodynamics are usually evaluated by vital signs, 12-lead ECG, transthoracic echocardiography, and administration of drugs such as catecholamine. Many donors show echocardiographic abnormalities and/or significant pressor/inotrope requirement. Abnormalities of the coronary arteries is usually checked under direct vision and the final decision of acceptance is made by the donor team.

Because no one can predict of the timing of donation, the transplant team must always be available in a short time. Furthermore, proximity of the donor and recipient teams is important because the permissible ischemic time for the heart is shorter than that for other organs.

\section{Donor Operation}

The final assessment of the donor heart is performed by visual inspection and palpation to check for contusion, recent ischemic events, heart disease and contractility after median sternotomy. Subsequently, the ascending aorta, pulmonary artery (PA), and superior and inferior venae cava (SVC, IVC) are dissected and preparation for cannulation for cardioplegia is performed in the ascending aorta. The donor heart team waits while the preparations are done by the other organ recovery teams. After completion of dissection of additional organs, heparin is given and the cardioplegia line is inserted into the ascending aorta and the cardioplegic solution is flushed. When the lungs are harvested at the same time, cannulation for pulmonary preservation is performed in the main PA by the pulmonary donor team. The SVC is tied and cut after infusion of pulmonary preservation solution from the PA. Subsequently, the ascending aorta is cross-clamped and pressurized cardioplegic solution is infused with cutting of the IVC.

In the bicaval method of recipient operation, the length of both the SVC and IVC is important. The site in the IVC is near the right atrium when the liver is harvested at the same time. The pulmonary vein and left atrium region are isolated with the pulmonary donor team. The SVC is cut right under the azygos vein and the ascending aorta is cut as long as possible. The PA is cut at the bifurcation and then the heart is excised, wrapped three times in plastic bags containing cardioplegic solution and transported within an 
icebox. Various types of cardioplegic solution can be used. We used St Thomas solution of the extracellular fluid type at first, but changed to Celsior solution from the $7^{\text {th }}$ case.

\section{Recipient Operation}

In the standard technique for HTx developed by LowerShumway, the left atrial anastomosis is performed first and then the right atrial anastomosis after creation of a right atrial cuff, which is created by an incision from the orifice of the IVC toward the right atrial appendage? In the bicaval method, the recipient right atrium is excised by transecting the SVC and the junction between the right atrium and IVC. The donor atria are preserved and left atrial anastomosis with a separate bicaval anastomosis is performed. The bicaval method has been modified to leave a strip of the posterior right atrial wall as a bridge connecting both venae cava by Kitamura et al6 In this method, the bridging posterior right atrium serves as a landmark of the right atrial cuff distorted by the caval snares. Adjustment of the length between the SVC and IVC is easy for a small donor heart because of the absence of shrinkage and retraction of the divided atrial cuffs (Figure 1).

A median sternotomy is performed. In patients with LVAS implantation, dissection of the heart must be done carefully because of severe adhesion, especially around the inlet and outlet cannulae including LV apex. The femoral vessels are prepared for emergency cannulation. Before the arrival of the donor heart, the recipient's heart and great vessels are exposed and prepared for cannulation. The recipient is heparinized and cannulation for cardiopulmonary bypass is performed. The site of aortic cannulation is distal to the anastomosis of the outlet cannula of the LVAS. For venous cannulation, right-angled cannulae are preferable because they do not obstruct the suturing of the bicaval anastomoses.

When the donor heart has arrived in the operating room, recipient heart excision is started with aortic cross-clamping and snaring of the SVC and IVC. An incision is made in the right atrium and extended to the coronary sinus. The aorta is divided just above the aortic valve and then the PA is divided just distal to the pulmonary valve. The left atrium is divided, starting at the septum and extending across the dome to the base of the left atrial appendage. The incision is continued to the mitral valve. Then the heart is removed. When a LVAS has been implanted, care must be taken to remove the anastomosis site of the outflow cannula in the ascending aorta. The inflow cannula is detached from the LV apex.

The donor heart is inspected for abnormalities, such as a patent foramen ovale (PFO), and valvular anomalies. If a PFO is found, it is closed from the right atrial side. The PA and aorta are separated. Next, the pulmonary vein openings are connected, creating a large left atrial cuff. In the LowerShumway method, the SVC is doubly ligated and the right atrium is opened from the IVC to the right atrial appendage without injuring the sinus node.

For the recipient anastomoses, the left atrial anastomosis is performed first. The donor left atrial cuff is sutured to the recipient's left atrium using a long 4-0 prolene suture from the level of the left superior pulmonary vein. A venting catheter is inserted in the left ventricle through the right pulmonary vein via the mitral valve. After completion of the left atrial anastomosis, the subsequent anastomoses are performed according to the ischemic time. Usually, the pulmonary anastomosis is performed after trimming to avoid excess length and possible kinking. This anastomosis is performed end-to-end with a running 5-0 prolene suture. Then the donor and recipient aortas are anastomosed endto-end with a running 4-0 prolene suture. The donor IVC is anastomosed to the recipient IVC-atrial cuff using a 5-0 prolene suture. After that, terminal cardioplegic solution is infused and the aortic cross-clamp is removed. The donor SVC and recipient SVC-atrial cuff are anastomosed using a 5-0 prolene suture.

Usually inotropic support such as dobutamine is given and the patient is weaned from cardiopulmonary bypass after confirming air removal and ventricular function by transesophageal echocardiography. Heart rate control is needed because the donor heart is denervated and a heart rate of 90-120 beats/min is maintained by infusion of isoproterenol or temporary pacing. Immunosuppression during the operation is performed by infusing $500 \mathrm{mg}$ of methylprednisolone twice: at the beginning of operation and just before declamping of the aorta.

In the case of LVAS implantation, the blood pump and cannulae are removed.

\section{Intensive Care After HTx}

The hemodynamic problems of transplant patients in the intensive care unit (ICU) at National Cardiovascular Center are summarized as denervated heart, transient cardiac dysfunction, pulmonary hypertension, and donor-recipient size mismatch? Cardiac pacing is applied to all patients and isoproterenol infusion is required in some cases to maintain an appropriate heart rate. Infusion of low-dose dopamine and atrial natriuretic peptide is performed for preservation of cardiac and renal function. Acute renal insufficiency occurred in 1 patient, who needed transient continuous hemodiafiltration. Early removal of catheter and tubes was attempted to reduce the risk of infection. In 1 case of donor-recipient size mismatch, cardiogenic pulmonary edema developed immediately after tracheal extubation, probably because of wound pain and afterload mismatch. Management of the denervated heart and preservation of renal function, in addition to conventional intensive care, are important in the intensive care of HTx patients.

\section{Management After HTx}

Important factors influencing the prognosis of HTx patients are primary graft failure, acute rejection, transplant coronary artery disease, infection, and malignancy.

\section{Primary Graft Failure}

Donor heart function, the preservation method, and the surgical method of harvesting affect graft function. If the donor has been infused with high doses of inotropic agents or has had an episode of cardiac arrest, donor heart function should be investigated carefully by electrocardiography and echocardiography. It is important to shorten the ischemic time of the donor heart as much as possible to preserve its function. As for cardioplegia, Celsior solution is easily available at donor hospitals. Transportation is another factor in shortening the ischemic time. Use of private jets or helicopter under direction of the JOTNW is desirable to ensure close communication between the donor and recipient teams.

\section{Acute Rejection}

Control of acute rejection is important and the results of 


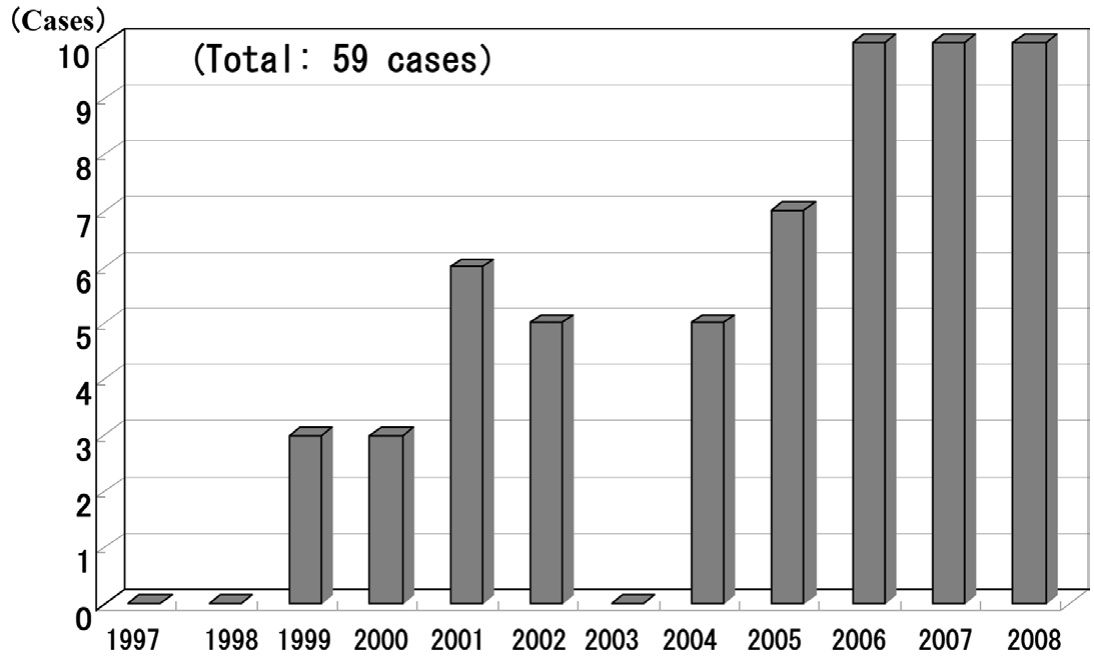

Figure 2. Year distribution of heart transplantation in Japan $!^{3}$
HTx have improved after adoption of a new immunosuppressive drug, cyclosporine, and development of the endomyocardial biopsy method from an internal jugular vein as the diagnostic procedure. Acute rejection is frequent within the first year and there are many asymptomatic cases. Therefore, scheduled endomyocardial biopsy is performed and the histopathologic judgment is based on the International Society for Heart and Lung Transplantation (ISHLT) standardized cardiac biopsy grading8

For immunosuppressive therapy, a 3-drug regimen including a calcineurin inhibitor (cyclosporine $\left(\mathrm{Neoral}^{\circledR}\right)$ or tacrolimus (Prograph $\left.{ }^{\circledR}\right)$ ) in combination with an antimetabolite (mycophenolate mofetil (Cellcept ${ }^{\circledR}$ ) or azathioprine) and steroid (predonine) is generally used. Induction therapy with monoclonal or polyclonal antibodies was also used in several cases. We have used, murine monoclonal CD3 antibody $\left(\mathrm{OKT} 3^{\circledR}\right.$ ) or IL-2 monoclonal antibody (basiliximab) in cases of severe renal dysfunction in the perioperative period.

The immunosuppressive agents are controlled by pharmacokinetic parameters, including trough level and the area under the plasma concentration curve?,10 ISHLT grade 3A or higher rejection is usually treated with steroid pulse therapy. If there is resistance to pulse therapy, cytolytic therapy consisting of monoclonal or polyclonal antibodies is instituted. Humoral or antibody-mediated rejection will occur if there is attacking of donor-specific antibodies! ${ }^{11}$ The panel reactive antibody test is useful for monitoring this, and plasmapheresis, intravenous immunoglobulin (IVIg), and rituximab (anti-B-cell antibodies) are used to treat humoral rejection.

\section{Transplant Coronary Artery Disease (TxCAD)}

TxCAD is thought to be chronic rejection of the allograft with the immunologic attack directed against the vascular endothelium of the allograft. Histologically, the intimal lesions are concentric, diffuse and extensive. Several factors, such as activation of $\mathrm{T}$ lymphocytes, antibodies, acute rejection, and cytomegalovirus (CMV) infection, are considered as possible etiologies, but the precise mechanism of TxCAD is still unclear.

Cardiogenic shock or sudden death from arrhythmia develops without chest pain because of denervation of the transplanted heart. We monitored TxCAD by routine coronary arteriography and intravascular ultrasonography at biopsy 3 months after transplant and at the annual checkup. Coronary artery bypass grafting, percutaneous transluminal coronary angioplasty or stenting are only palliative because of the diffuse nature of TxCAD. Recently, the proliferation signal inhibitors or mammalian target-of-rapamycin inhibitors, everolimus and sirolimus, demonstrated a benefit in reducing maximal intimal thickness and subsequently TxCAD in de novo HTx recipients ${ }^{12}$ Everolimus was introduced to Japan in 2007, and is used for patients with TxCAD. Long-term follow-up of these patients is essential.

\section{Infection}

Infection is the leading cause of death of HTx recipients during the $1^{\text {st }}$ year after transplant, according to the ISHLT registry4 Bacterial infection is common during the 1st month after transplant and subsequently, opportunistic infections, such as CMV and herpes simplex virus, increase. CMV infection is considered to be a risk factor of TxCAD, so at the National Cardiovascular Center, CMV-PCR is performed routinely. In the management of infection, care should be taken to prevent it and to treat it early.

\section{Malignancy}

Malignancy, including lymphoma, is the cause of death in over $20 \%$ of HTx recipients who survive more than 3 years from JSHLT registry. Lymphoma (post-transplant lymphoproliferative disorder: PTLD) is a common tumor after transplantation and it is speculated that it might be related to the overall level of immunosuppression, especially the calcineurin inhibitor. Epstein-Barr virus (EBV) has a possible causative role in PTLD, so we perform check of EVB-PCR at routine follow-up visits.

\section{Long-Term Follow-up After HTx}

Consideration must be given to hypertension, renal dysfunction, hyperlipidemia, diabetes mellitus to maintain the quality of life of HTx recipients. Adjustment of immunosuppressive drugs is important to reduce side-effects, and early detection of infection, malignancy, and TxCAD improves prognosis.

\section{Results Worldwide}

According to the ISHLT registry of 2008 , over 80,000 HTx have been performed worldwide. Between January 


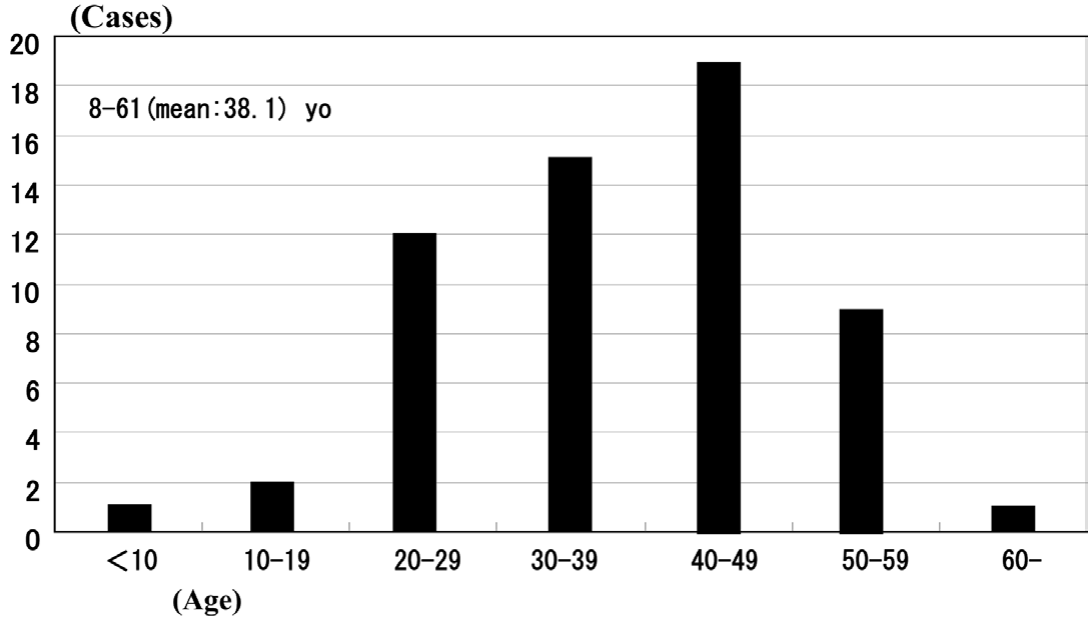

(cases)

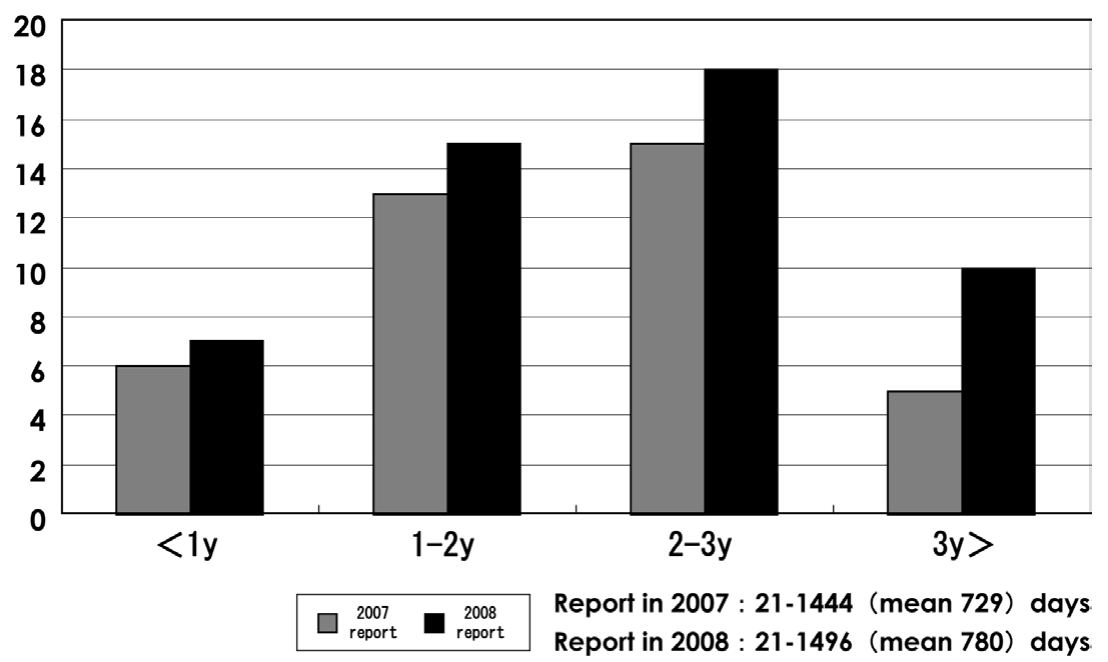

Figure 3. Age distribution of heart transplant recipients in Japan ${ }^{3}$

Figure 4. Duration of support with a left ventricular assist system in heart transplant patients in Japan! ${ }^{13}$
1982 and June 2006, 74,267 HTx were performed and survival rates were $81.6 \%$ at 1 year, $68.6 \%$ at 5 years, $50.6 \%$ at 10 years, and $19.6 \%$ at 20 years. Recent survival rates have improved compared with the early phase of the registry (at 5 years (1982-1991): 64.6\%; 70.0\%, 1992-2001; 72.3\%, 2002-2006). Survival decreases at a linear rate of approximately $3.5 \%$ per year. For immunosuppressive therapy, the combination of cyclosporine (or tacrolimus), mycophenolate mofetil, and predonine is the most common, although recently, tacrolimus has overtaken cyclosporine as the most common calcineurin inhibitor. Sirolimus is used in over $10 \%$ of recipients at 1 -year post transplant. As an induction therapy, use of interleukin-2 receptor antagonist is increasing. According to the functional status reported on the 1-, 3-, 5- and 7-year annual follow-up (between 1995 and June 2006), approximately $90 \%$ of survived recipients have no activity limitations up to 7 years after transplant.

\section{Results in Japan}

Among the 333 candidates for HTx registered with the JOTNW from October 1997 to September 2008, 59 HTx were performed in 7 institutes (National Cardiovascular Center: 24; Osaka University: 17; Tokyo University: 6; Tokyo Women's Medical University: 4; Saitama Medical

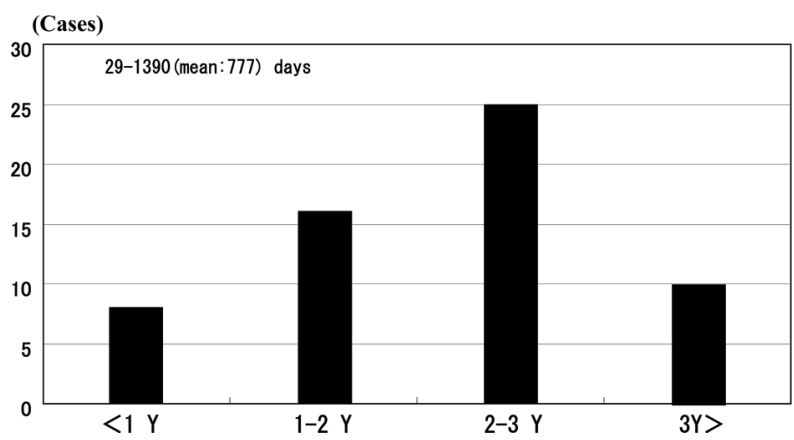

Figure 5. Waiting period for heart transplantation in Japan ${ }^{13}$

University: 3; Kyusyu University: 3; Tohoku University: 2) ${ }^{13}$ (Figure 2). Of these cases, the first was performed at Osaka University ${ }^{14}$ and subsequently 2 cases were performed at the National Cardiovascular Center in 1999!5 However, 35 candidates underwent HTx abroad and 109 died while on the waiting list; 118 candidates are awaiting HTx and of those, $51 \%$ are status $1,42 \%$ are status 2 , and $7 \%$ are status 3. The main characteristic of the transplanted patients was DCM as the major primary indication for HTx, and IHD was present in 3 of the 59 (5\%). In the ISHLT registry, IHD 
Survival rate $(\%)$

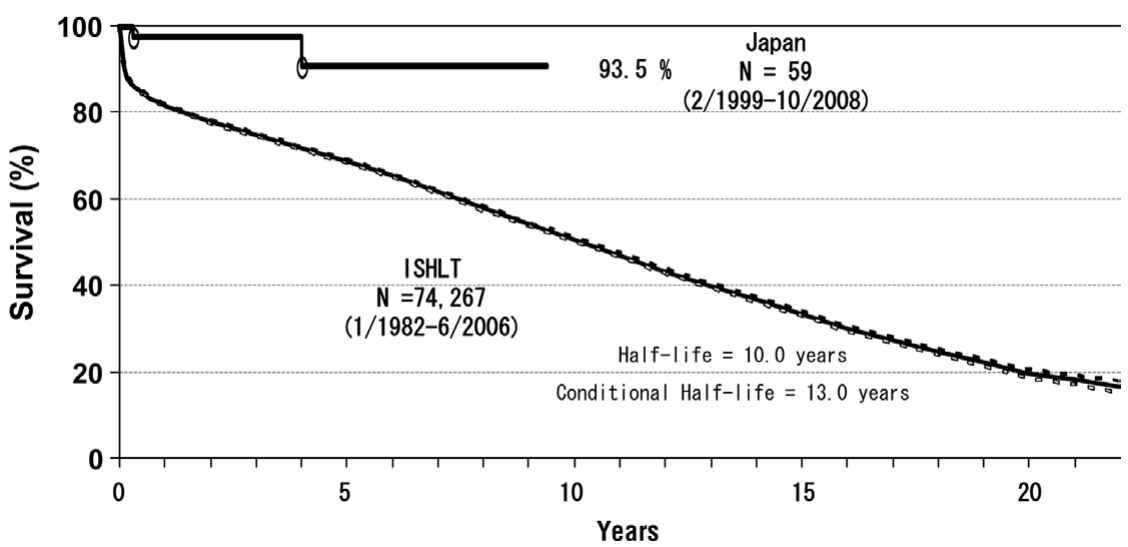

Figure 6. Kaplan-Meier survival for heart transplantation worldwide and in Japan.,13 is the indication in approximately $30 \%$ of recipients. In contrast, over $90 \%$ of the Japanese HTx recipients did not have IHD. The age distribution of the recipients was between 8 and 61 (mean 38.1) years old (Figure 3). Male patients comprised 35 cases and females 17 cases. All recipients were status 1 , including 9 with inotropic support in the ICU/CCU. The other 50 patients (84.7\%) had several types of LVAS: 36 received the Toyobo LVAS (34: left ventricular drainage, 2: left atria drainage), 10 had an implantable pulsatile LVAS (Novacor: 5, HeartMate: 5), and the other 4 had an implantable non-pulsatile LVAS. Support duration was between 21 and 1,496 days (mean 780 days) (Figure 4). Waiting period as status 1 was between 29 to 1,390 days (mean 777 days) (Figure 5). The waiting time has recently extended; only 8 cases underwent HTx within 1 year of listing on the JOTNW, and 10 cases have been waiting for more than 3 years.

With regard to the cardioplegic solution, Celsior, Modified Collins solution, St Thomas solution, Bredshnieders solution, and UW solution were used initially, but now Celsior solution is mainly used. For the recipient operation, initially the Lower-Shumway method was used, but the bicaval method was used in several cases and then the modified bicaval method was developed and used in many cases (68\%). In 1 case, aortic valve replacement was performed simultaneously. The majority of patients underwent the modified bicaval method with Celsior solution for cardiac preservation, and $64.4 \%$ of recipients were administered triple therapy with cyclosporine, mycophenolate mofetil, and steroid as the initial immunosuppressive regimen. Recently, tacrolimus has been used instead of cyclosporine. Induction therapy was performed in 19 cases. From last year, basiliximab (Simulect ${ }^{\circledR}$ ) was used as induction therapy in some cases.

Only 2 patients died after HTx (pneumonia: 4 months after HTx; infection: 50 months after HTx). The longest survival is up to 10 years after HTx. The 5-year survival rate is $93.5 \%$, which is superior to that of the international registry (Figure 6).

\section{Future Prospects}

HTx in Japan has been very limited by a severe shortage of donors, but the results have beene excellent even though the majority of recipients had a LVAS as a bridge to HTx. Progress of the HTx program, including children ${ }^{16}$ in Japan is expected.

\section{References}

1. Barnard CN. A human cardiac transplant: An interim report of a successful operation performed at Groote Schuur Hospital, Cape Town. S Afr Med J 1967; 41: 1271-1274.

2. Cooley DA, Bloodwell RD, Hallman GL, Nora JJ, Harrison GM, Leachman RD. Organ transplantation for advanced cardiopulmonary disease. Ann Thorac Surg 1969; 8: 30-46.

3. Caves PK, Stinson EB, Billingham ME, Shumway NE. Serial transvenous biopsy of the transplanted human heart: Improved management of acute rejection episodes. Lancet 1974; 1: 821-826.

4. Taylor DO, Edwards LB, Aurora P, Christies JD, Dobbels F, Kirk R, et al. Registry of the international society for heart and lung transplantation: Twenty-fifth official adult heart transplant report 2008. J Heart Lung Transplant 2008; 27: 943-956.

5. Lower R, Stofer RC, Shumway NE. Homovital transplantation of the heart. J Thorac Cardiovasc Surg 1961; 41: 196-204.

6. Kitamura S, Nakatani T, Bando K, Sasako Y, Kobayashi J, Yagihara T. Modification of bicaval anastomosis technique for orthotopic heart transplantation. Ann Thorac Surg 2001; 72: 1405-1406.

7. Imanaka $\mathrm{H}, \mathrm{Nakatani} \mathrm{T}$, Kitamura S. Complications and problems of acute care in patient undergoing heart transplantation. Kyobugeka 2007; 60: 958-962 (in Japanese).

8. Billingham ME, Cary NR, Hammond ME, Kemnitz J, Marboe C, McCallister HA, et al. A working formulation for the standardization of nomenclature in the diagnosis of heart transplantation and lung rejection: Heart rejection study group. J Heart Lung Transplant 1990; 9: $587-593$.

9. Wada K, Takeda M, Ueda T, Ochi H, Kotake T, Morishita H, et al. Relationship between acute rejection and cyclosporine or mycophenolic acid levels in Japanese heart transplantation. Circ J 2007; 71: 289-293.

10. Wada K, Takeda M, Kotake T, Ochi H, Morishita H, Komamura K, et al. Limited sampling strategy for mycophenolic acid in Japanese heart transplant recipients: Comparison of cyclosporin and tacrolimus treatment. Circ J 2007; 71: 1022-1028.

11. Kobashigawa J, Mehr M, West L, Kerman R, George J, Rose M, et al. Report from a consensus conference on the sensitized patient awaiting heart transplantation. J Heart Lung Ttransplant 2009; 28: 213-225.

12. Zuckermann A, Manito N, Epailly E, Fiane A, Bara C, Delgado JF, et al. Multidisciplinary insights on clinical guidance for the use of proliferation signal inhibitors in heart transplantation. $J$ Heart Lung Transplant 2008; 27: 141-149.

13. The Japanese Society for Heart Transplantation. The registry report of Japanese heart transplantation 2008. Ishoku 2008; 43: 470-473 (in Japanese).

14. Matsuda H, Fukushima N, Sawa Y, Nishimura M, Matsumiya G, Shirakura R. First brain dead donor heart transplantation under new legislation in Japan. J Jpn Thorac Cardiovasc Surg 1999; 47: 499505 .

15. Kitamura S, Nakatani T, Yagihara T, Sasako Y, Kobayashi J, Bando $\mathrm{K}$, et al. Cardiac transplantation under new legislation for organ transplantation in Japan: Report of two cases. Jpn Circ J 2000; 64: $333-$ 339.

16. Kobayasihi N, Ono Y, Tsukano S, Yazaki S, Yamada O, Echigo S, et al. Outcome of pediatric candidates for orthotopic heart transplantation at National Cardiovascular Center in Japan. Pediatr Cardiol Card Surg 2008; 24: 628-635 (in Japanese). 